\title{
Elongated Silicon-Carbon Bonds at Graphene Edges
}

Qu Chen ${ }^{l}$, Alex W. Robertson ${ }^{l}$, Kuang He ${ }^{l}$, Chuncheng Gong ${ }^{l}$, Euijoon Yoon ${ }^{2}$, Angus I.

Kirkland $^{l}$, Gun-Do Lee ${ }^{2 *}$, Jamie H. Warner ${ }^{1 *}$

${ }^{1}$ Department of Materials, University of Oxford, Parks Road, Oxford, OX1 3PH, United

Kingdom

${ }^{2}$ Department of Materials Science and Engineering, Seoul National University, Seoul, 151-742, Korea

*jamie.warner@materials.ox.ac.uk; gdlee@snu.ac.kr;

S1. Elemental Identification of Si Atoms on Graphene using Energy Dispersive X-Ray Spectroscopy 


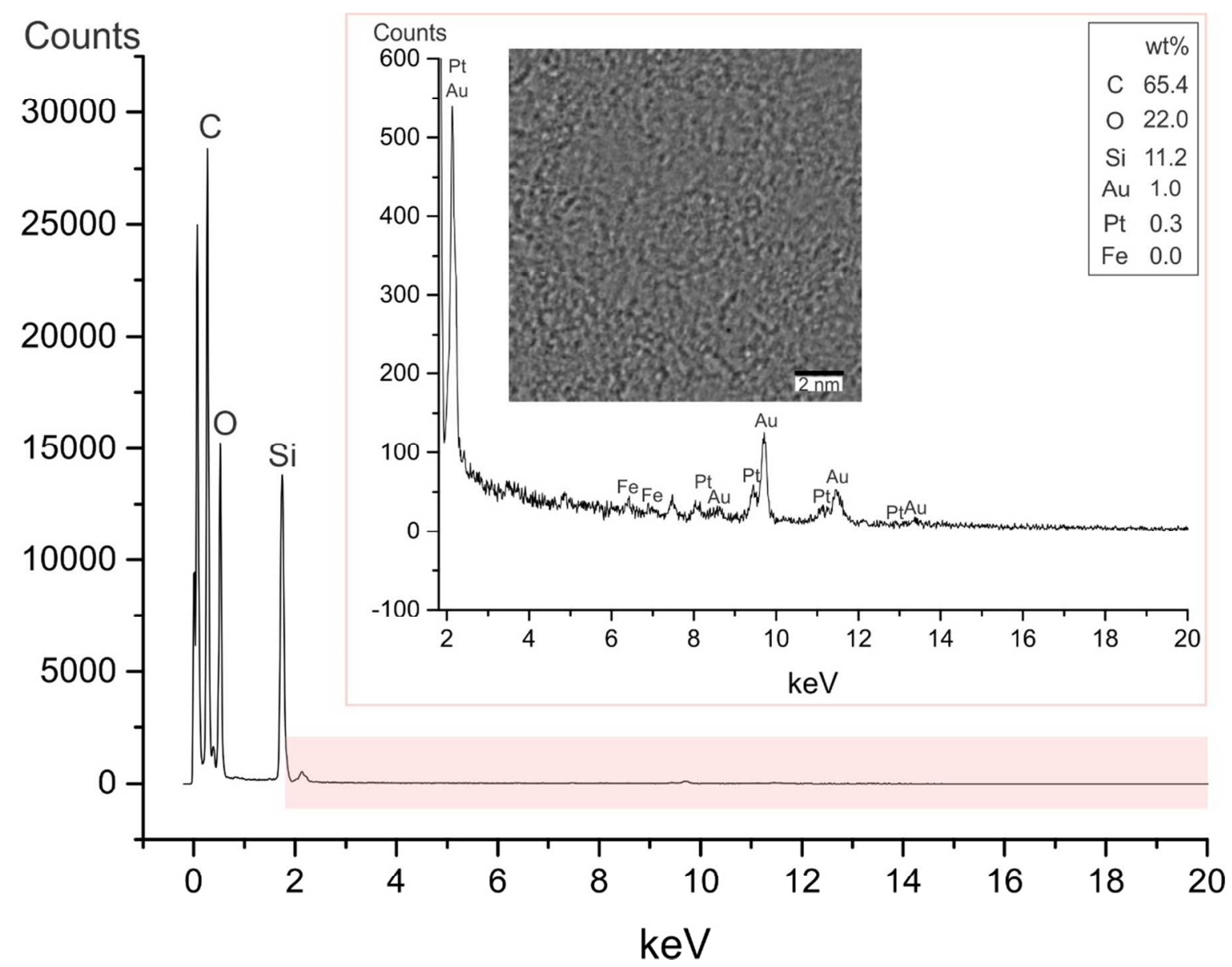

Figure S1 Low magnification image and EDX spectrum, and magnifying spectrum showing detailed peaks from $2 \mathrm{keV}$ to $20 \mathrm{keV}$.

According to the EDX spectrum in Figure S1, there are four kinds of foreign elements, $\mathrm{Si}, \mathrm{Au}, \mathrm{Pt}$ and $\mathrm{Fe}$, presence within the graphene sample with various weight percentage, and they can attach to a graphene edge within a hole. However, with consideration of their relative content and the atomic masses, a mole ratio of $\mathrm{Si}$ to $\mathrm{Au}$ to $\mathrm{Pt}$ is $260: 3.3: 1$, indicating that the possibility for observing a Si atom is far higher than an Au or Pt atom (the weight percentage of Fe is lower than $0.1 \%$ thus can be neglected). Therefore, it is highly probably that the foreign atoms observed at the graphene edges are predominantly Si atoms. The different atomic mass of Si and 
$\mathrm{Au}$ also gives rise to different contrast relative to the carbon lattice, making their identification also possible based on contrast. 


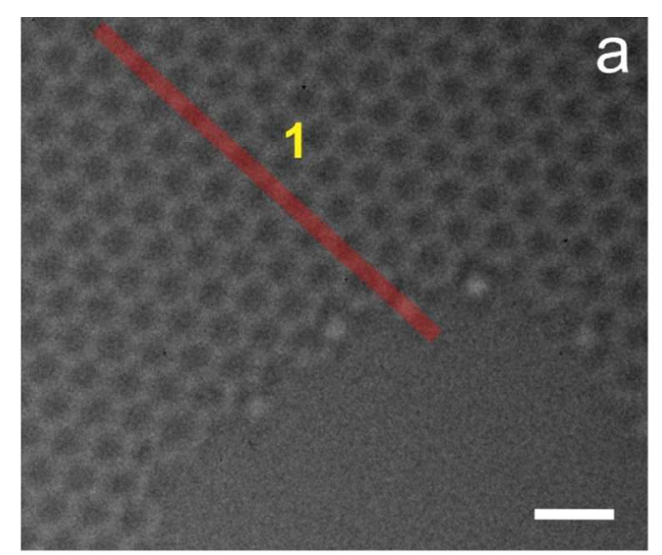

C
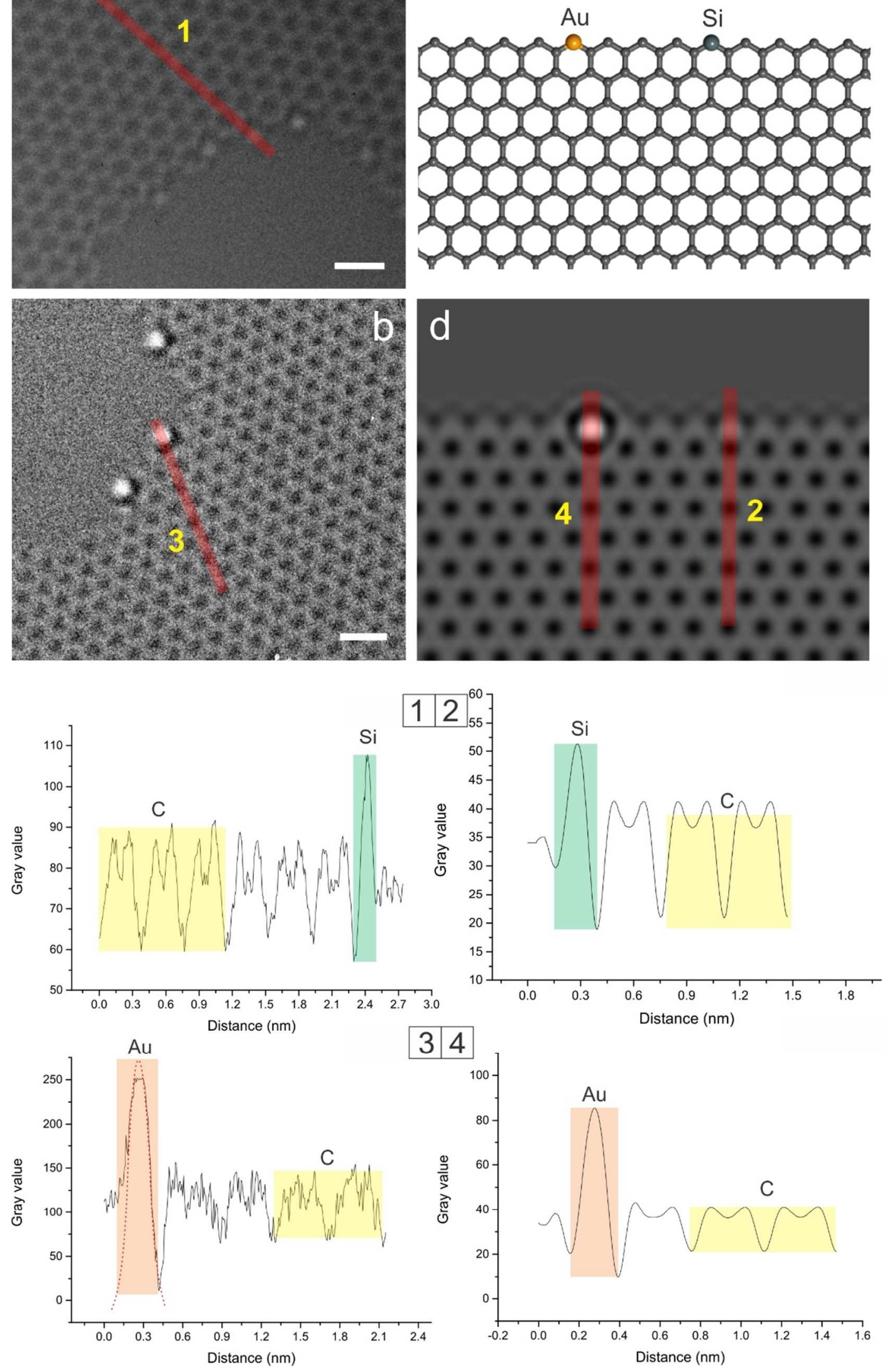
Figure S2 Comparison of contrast ratios for single $\mathrm{Si} / \mathrm{Au}$ atoms to $\mathrm{C}$ atoms. a) AC-TEM image with $\mathrm{Si}$ atoms sitting at the graphene edge. b) AC-TEM image with Au atoms sitting at graphene edge. c,d) Atomic model and its image simulation of a Si atom and a gold atom at graphene edge. Red rectangles 14 represent the region where box-average intensity profiles are measured (intensity profiles 1-4). Coloured box in the intensity profiles demonstrate the contrast for different elements. Scale Bar: $0.5 \mathrm{~nm}$. Intensity information in AC-TEM images can be considered as a supplementary evidence for the elemental identification. Figure S2a is AC-TEM image of a graphene hole with several noncarbon atoms attached, and its box-average intensity profile indicates a contrast ratio for the noncarbon atom to carbon atom of about 1.5-1.6. It has been reported by Robertson et al that $\mathrm{Si} / \mathrm{C}$ contrast ratio is approximately 1.5 , with the standard deviation of about $\pm 0.2 .{ }^{1}$ Occasionally, we also observed some graphene holes with atoms attached that have significantly higher contrast spots, as shown in Figure $\mathrm{S}$ b. The single atoms possess much higher atomic mass than $\mathrm{Si}$, and based on the EDX results, gold atoms are the likely element. The contrast ratio in this case is about 3.5 based on the intensity profile 3 . An atomic model with an isolated Si atom and Au atom attached to a graphene edge (Figure S2 b) is used for a multislice image simulation (Figure S2d), and comparisons of the contrast ratios of a single Si atom and single Au atom to the C atom (intensity profile 2 and 4), are 1.56 and 3.68 respectively. These simulation results agree well with the values obtained from TEM images, further confirming that the single dopant atoms at graphene edges we are observing in most of the TEM images are Si atoms. We excluded all atoms that showed contrast associated with gold atoms in our analysis within the main text.

It is worth mentioning that highly stable Si atoms are carefully chosen when evaluating the $\mathrm{Si} / \mathrm{C}$ contrast ratio as the contrast can be misleading if the $\mathrm{Si}$ atom migrates during the acquisition time period, for example, in figure 6 in the main text. The Si atoms with the EK edge 
configuration are relatively mobile compared to the former configurations from figure 1 to 5 , thus it can be seen that the $\mathrm{Si} / \mathrm{C}$ contrast is slightly lower than the value it is supposed to be, and the various contrasts are determined by the steadiness of the Si atoms.

\section{S2. Stability of the Two Most Frequently Appearing Si-Graphene Edge Configurations}
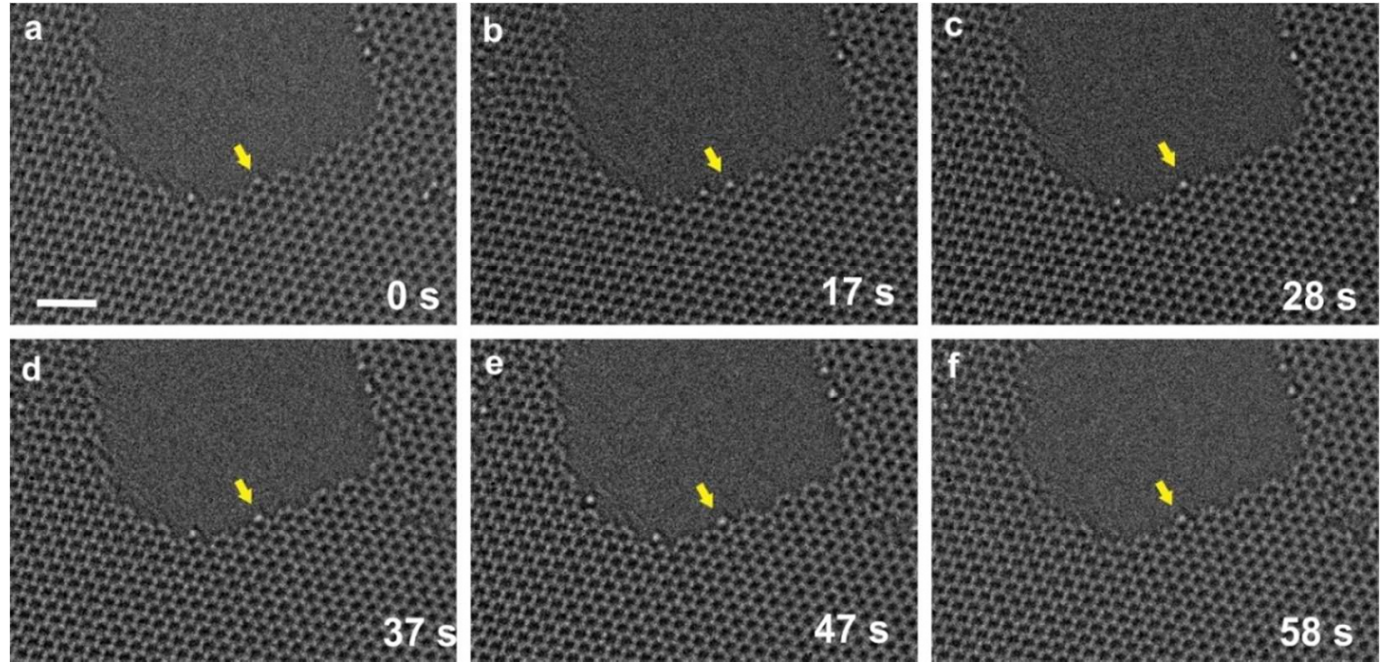

Figure S3 A Si atom at pentagon configuration (pointed by yellow arrows) in 6 continuous frames.
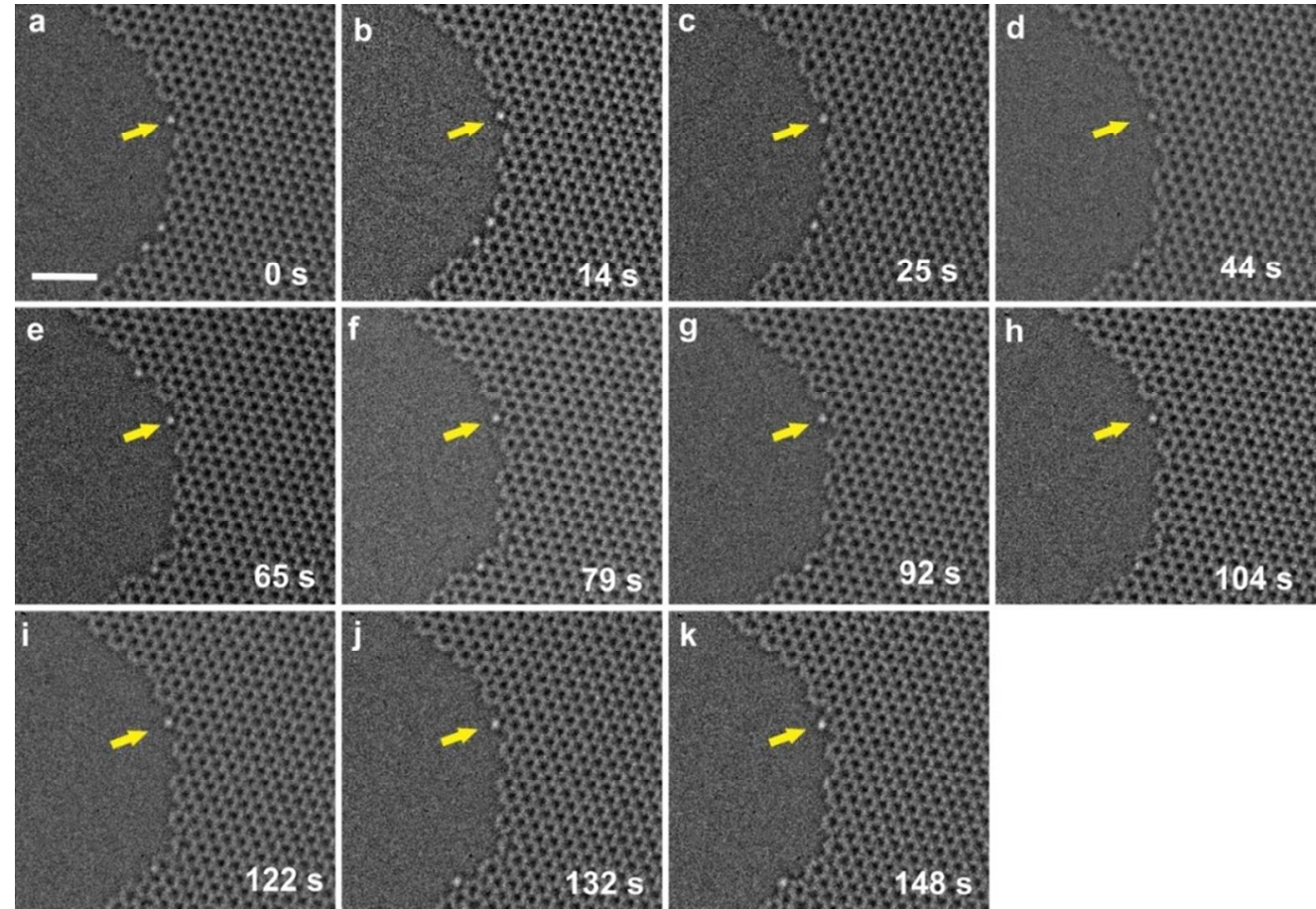
Figure S4 A Si atom at tetragon configuration (pointed by yellow arrows) in 11 continuous frames.

\section{S3. Fully Relaxed DFT Calculated Model for the Three-fold Coordinated Si Atom at Graphene Edge}

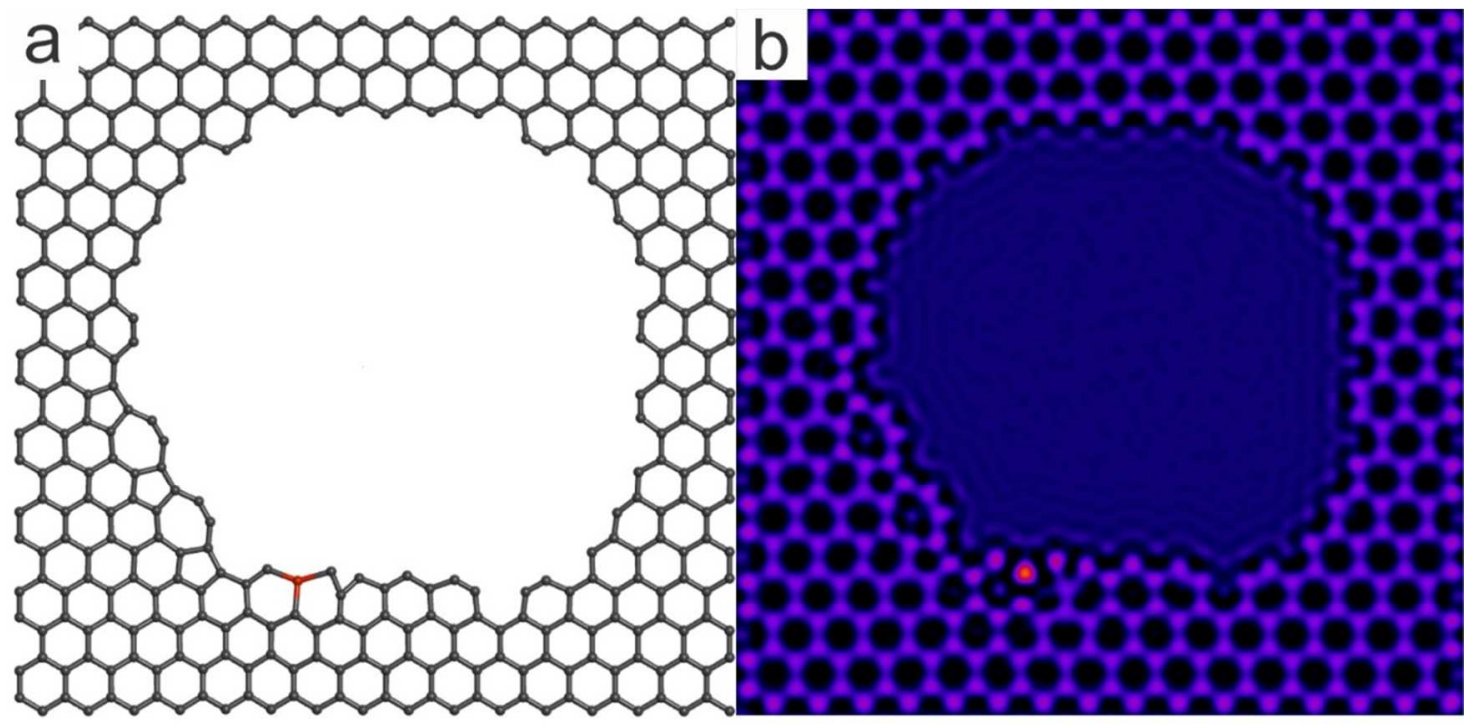

Figure S5 a)The fully relaxed model calculated by DFT showing a three-fold coordinated Si dopant (red) at the edge of a graphene hole. This model is built with the consideration of the surrounding environment of the $\mathrm{Si}$ atoms, including the 5-7 reconstructed edge and one absent $\mathrm{C}$ atom. b) Multislice image simulation of a). The part of the model and the simulation image is shown in Figure $4 \mathrm{e}$ and $4 \mathrm{f}$ in the main text.

\section{S4. Bond Length Measurement with the Presence of Astigmatism in TEM Images}

The accuracy of measured bond length values is strongly determined by the degree of astigmatism of a TEM image. The regular hexagonal rings of graphene lattice is slightly distorted, which also affected the shape in reciprocal space. In this occasion, the scale is the function of direction. In addition, the astigmatism is not uniform within a whole TEM frame, 
therefore selecting the region for scale setting with care is essential for precise measurement of a bond distance.

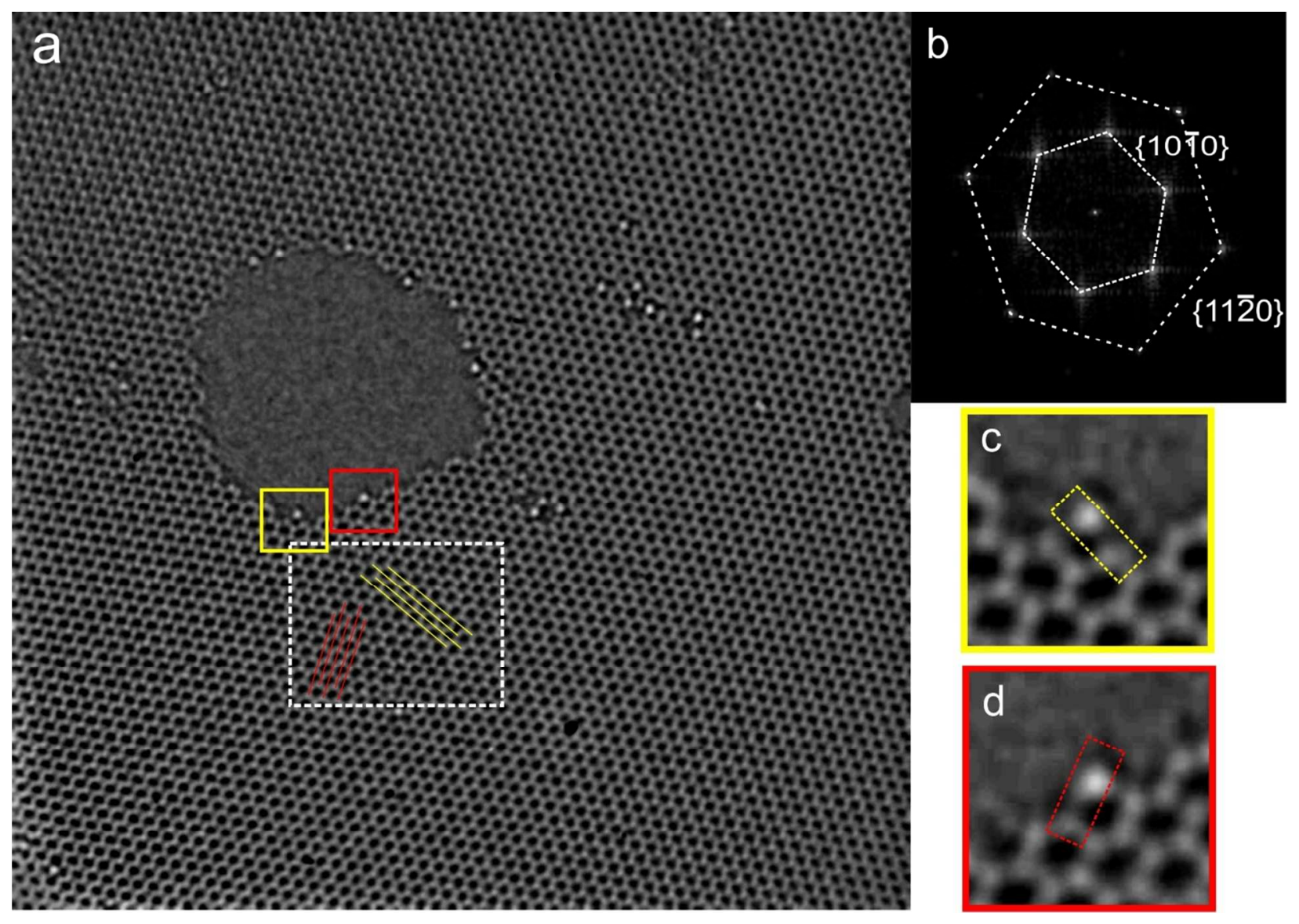

Figure S6 a) AC-TEM image with Si atoms at graphene edge. b) Fast Fourier Transform (FFT) pattern for the region in white dashed box in a). c) Magnifying image of the yellow box (tetragonal configuration) in a) with a Si-C bond highlighted. d) Magnifying image of the red box (pentagonal configuration) in a) with a Si-C bond highlighted.

Figure S6 provides an example for the measurement of Si-C bond lengths in two Si-graphene edge configurations: tetragonal configuration (yellow box) along zigzag edge and pentagonal configuration (red box) along armchair edge with the consideration of the effect of astigmatism. The region in the white dashed box in Figure S6a is selected to be the referential C-C bonds for 
scale setting, as it is a pristine graphene area (barely with defect) close to the targeted Si-C bonds. The corresponding FFT pattern is in Figure S6b. It can be observed that neither the six spots for $\{10 \overline{1} 0\}$ planes nor those corresponding to $\{11 \overline{2} 0\}$ planes is regular hexagon, indicating the presence of astigmatism, which means setting scale based on the FFT pattern is no longer feasible (C-C bonds in graphene are not perpendicular to the crystal planes). To obtain the actual Si-C bond distances, the key is to know the average $\mathrm{C}-\mathrm{C}$ bond length parallel to the $\mathrm{Si}-\mathrm{C}$ bonds:

$$
\mathrm{Si}-\mathrm{C} \text { bond length in } \mathrm{pm}=\frac{S i-C \text { bond length in pixel }}{\text { average } C-C \text { bond length in pixel }} \times 142 \mathrm{pm}
$$

For instance, to obtain the Si-C bond length in Figure S5c, we need to know the average C-C bond length along the direction highlighted by yellow lines in Figure S5a. 50 bond lengths in the white dashed region along that direction were manually measured in pixel:

$$
\text { Si }-\mathrm{C} \text { bond length }=\frac{26.39}{20.12} \times 142 \mathrm{pm}=186 \mathrm{pm}
$$

The standard deviation of the $50 \mathrm{C}-\mathrm{C}$ bond lengths is $1.9 \%$, which is far smaller than the difference between Si-C and C-C bond distance. Same procedure was applied to the Si-C bond in Figure S5d and all the other Si-C bonds in the main text.

\section{S5. Si-C Bond Lengths at Room Temperature}

At room temperature, a graphene hole expands rapidly due to the chemical etching effects related to oxygen and hydrogen gas molecules in the vacuum. This causes Si atoms to be highly mobile around the graphene edges and makes their high resolution imaging more challenging that at elevated temperatures. At the elevated temperatures, main text results, chemical etching is reduced and the Si atoms remain stable in fixed positions for longer and this helps obtain high quality images of their atomic structure. Figure S7 shows two AC-TEM images taken at room 
temperature with a Si atom at the crest of zigzag edge, i.e., the two-fold coordinated substitutional position. This configuration is the same as the high-temperature counterpart shown in Figure 5. The average Si-C bond length at room temperature is $178 \pm 10 \mathrm{pm}$, similar to the same structure at $800{ }^{\circ} \mathrm{C}$. This suggest that temperature might not impact on the Si-C bond distances within the accuracy of our AC-TEM image resolution.
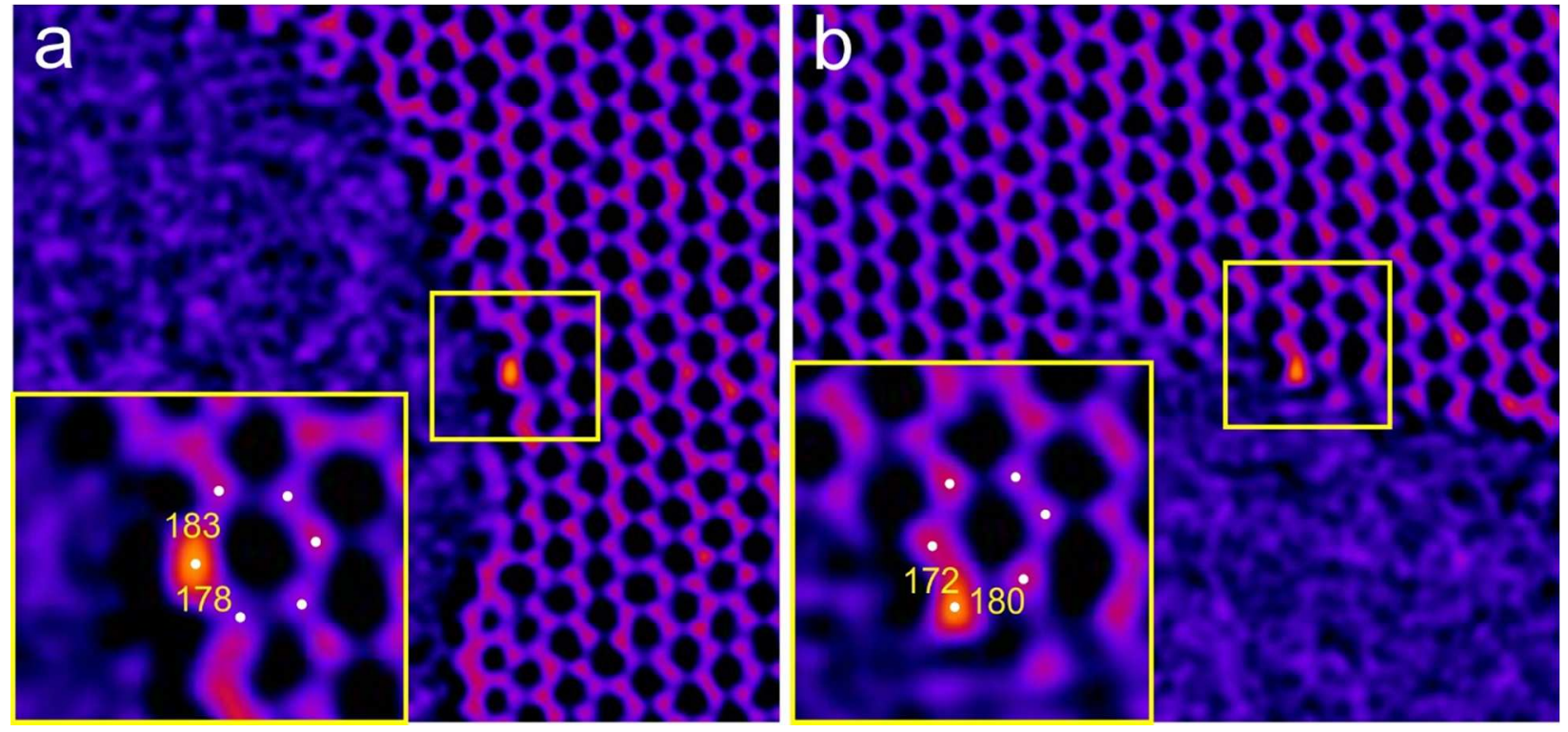

Figure S7. (a) and (b) Two AC-TEM images of Si atoms bonded to graphene edges at room temperature. Elongated bonds are detected in both cases. 